\title{
REAL-TIME DETECTION OF VOLATILITY IN LIQUIDITY PROVISION
}

\section{MATT BRIGIDA ${ }^{*}$}

1. SUNY Polytechnic Institute, Utica, USA

* Corresponding Author: Matt Brigida, SUNY Polytechnic Institute, 100 Seymour Rd., Utica, NY 13502 $\triangle$ matthew.brigida@sunypoly.edu

\begin{abstract}
Previous research has found that high-frequency traders will vary the bid or offer price rapidly over periods of milliseconds. This is a benefit to fast traders who can time their trades with microsecond precision, however it is a cost to the average market participant due to increased trade execution price uncertainty. In this analysis we attempt to construct real-time methods for determining whether the liquidity of a security is being altered rapidly. We find a four-state Markov switching model identifies a state where liquidity is being rapidly varied about a mean value. This state can be used to generate a signal to delay market participant orders until the price volatility subsides. Over our sample, the signal would delay orders, in aggregate, over 0 to $10 \%$ of the trading day. Each individual delay would only last tens of milliseconds, and so would not be noticeable by the average market participant.
\end{abstract}

Keywords: High-Frequency Trading; Liquidity; Markov-Switching Models

JEL: G10; G12; C24; C45

\section{Introduction}

The goal of this analysis is to construct methods to determine, in real-time, when the volatility of the liquidity provided is being rapidly changed around a mean value, which is consistent with the effect of an algorithm or set of algorithms. Such methods would allow the creation of orders which can be cancelled, or delayed, if the market switches to such a regime with unstable liquidity. This is analogous to the crumbling quote signal from the Investors Exchange (outlined in Bishop (2017)).

Such real-time detection is a difficult task, though identification does not have to be perfect. The threshold is that investors choose to use the order-that it is correlated enough with undesirable activity that it adds value to the investor to submit the order type. For the order type to have worth to investors algorithmic activity, or other processes which rapidly change liquidity around a mean value, which is a cost to the average investor must exist.

Hasbrouck (2018) found evidence for substantial volatility in the bid and offer prices which was not due to fundamental changes in the asset value. The cost of this volatility is not borne equally by traders. Faster traders are able to choose the point (in microseconds) at which they trade. Slower traders, however, will receive a trade price some time later (maybe seconds) after they attempt to submit a marketable order. This trade price is a random variable, and they are exposed to price risk which is a function of the expected variation of the bid (or offer) price over the time from when they submitted the order to when it is matched by the exchange. 
So, when fast traders change the bid/ask price quickly, slower traders still expect to receive/pay the same amount for each sell/buy order, however they have increased uncertainty. This increased risk without increased compensation should be avoided by any rational investor. The goal of our analysis is to help investors find ways to delay their order until the execution price of their order has more certainty. Since the volatility can occur in milliseconds, the method of identification must itself be algorithmic.

Note, investors should attempt to avoid these periods of increased uncertainty even if the source of the uncertainty is not high-frequency traders. We therefore don't attempt to determine the source of uncertainty, but rather, in real time, identify when such variations in liquidity are occurring.

Both spread and depth pose substantial risk, particularly for institutional investors who tend to trade in quantities far larger than what is available at the inside quotes. Despite this many seminal models of market making under asymmetric information ignore market depth by assuming a unit size for all trades (Copeland and Galai (1983); Glosten and Milgrom (1985); Easley (1992)). Alternatively, in Kyle (1985) market depth is implicitly incorporated in the model through requiring specialists to supply complete pricing functions. In our analysis we will consider the time-series of liquidity available in the orderbook within a set distance from the bid-ask midpoint.

Our algorithm will attempt to filter out the other various drivers of price and market depth changes. For example, French and Roll (1986) found evidence that stock price volatility is driven by private information being incorporated into market prices via trading. Lee, Mucklow, and Ready (1993) studied the relationship between spreads and depth around earnings announcements. So, we are attempting to find a state where price and market depth are changing in a manner inconsistent with trading on private information or around events. Notably, this first source of price and depth change would impart a directional bias to prices, and in the case of Lee, Mucklow, and Ready (1993) the spread widened. Alternatively, the high-frequency trading we are attempting to identify does not change mean price or market depth as in these former cases.

\section{Data}

We use data for the heavily traded E-Mini S\&P 500 Futures contract. Price discovery in the equity market occurs in this contract (Hasbrouck (2003)). Trading hours from Sunday-Friday from 6:00 p.m. to 5:00 p.m. Eastern Time (ET). Contract value is $\$ 50$ times the futures price. Cash delivery with expirations every 3 months. Traded on the Chicago Mercantile Exchange (CME) (pit and electronic (Globex)).

The reason we use CME Data ES is because, in addition to being the first place that information is incorporated into prices and trading overnight, all trades and quotes take place in this one central book. So, there is no delay in orders due to location.

Data are Market Depth Data' for E-Mini S\&P 500 futures (Globex), for the trading week from November 7 to November 11, 2016. The data were purchased directly from the CME. We focus our results on November 92016 because it was the trading day where results of the US Presidential election were released, and therefore there were high levels of trade and quote volume, which makes the presence of algorithmic activity more likely. 
Market Depth Data contains all market messages (trade/limit order updates) to and from the CME, and is time-stamped to the nanosecond. The data also includes tags for aggressor side. Using this data, we can recreate the ES orderbook with nanosecond resolution and up to 10 levels deep. The data are encoded in the CME's FIX/FAST message specification². We have made the translation scripts used in this analysis freely available ${ }^{3}$.

In the following charts and analysis, it is helpful to note the difference between clockand markettime. When considering the nanosecond (one-billionth of a second) level, the market has long periods of inactivity interspersed with periods of activity. Our data set only contains these periods of activity (and of course the length of time since the previous period of activity). Otherwise we would require a time series of 1 billion data points to analyse each second.

\section{Methods}

Our challenge is that of unsupervised learning - we are attempting to identify a state without training data providing the states for a sample of data. A classic problem of this type in the economics literature is to determine if the economy is in an expansion or recession. In this expansion/recession analysis Markov regime-switching regressions are used (see for example the method employed by the US Federal Reserve). We'll use a similar approach in our analysis to determine periods of stable, and unstable, liquidity driven by algorithmic activity. Our exact model is outlined below.

We measure liquidity on each side of the book as the amount of ES that can be bought within one point of the present bid-offer midpoint. One point is equivalent to 4 ticks (so maximum the inside quote and 3 additional levels of the book). Results below are for the November 9, 2016 trading day, which is the most likely to exhibit algorithmic trading activity due to the large public release of information, and the consequent portfolio rebalancing and increased trade volume.

\subsection{Markov-Switching Model}

There is no test for the proper number of states in a multiple state model. We thus estimate an increasing number of states and let the interpretation of the results and standard tests of the residuals, in each state, to guide us to finding a state consistent with algorithmic activity.

The two-state version of our model is:

$$
\begin{aligned}
& L i q_{t}= \begin{cases}\alpha_{1}+\beta_{11} L i q_{t-1}+\beta_{12} \Delta B A M+\epsilon_{1}, & \epsilon_{1} \sim N\left(0, \sigma_{1}\right) \\
\alpha_{2}+\beta_{21} L i q_{t-1}+\beta_{22} \Delta B A M+\epsilon_{2}, & \epsilon_{2} \sim N\left(0, \sigma_{2}\right)\end{cases} \\
& P\left(s_{t}=j \mid s_{t-1}=i\right)=p_{i j} \text { for } i, j \in\{1,2\} \text { and } \sum_{j=1}^{2} p_{i j}=1
\end{aligned}
$$

where Liq $_{t-1}$ is the liquidity in the previous period and $\triangle B A M$ is the most recent change in the bid-ask midpoint. There are two states, denoted by $s_{1}$ and $s_{2}$, and $p_{i j}$ denotes the probability that the state is $j$ given the state was $i$ in the previous period. We estimate the model via the 
Hamilton Filter with a custom implementation in $C++$ due to the large number of points in our time series.

Similar to the bid and ask volatility estimate in Hasbrouck (2018), we estimate the model for the bid and ask sides of the book separately. This is because the rapid deviations from a mean liquidity value, which we are attempting to identify, largely affect one side of the book, and so are more likely to be an artifact of the trading process rather than due to fundamental information. Nonetheless, modelling the entire book (bid and ask sides jointly) would include more information in the parameter estimates, such as spillover effects. However, this would increase the time required to estimate parameters as well as the time it takes to create a state prediction. Since the algorithm must be very quick to be useful, we err on the side of speed relative to the benefit of the information in both sides of the spread.

\subsubsection{Two States}

The two-state model is picking up states of changing liquidity and stable liquidity. In both the bid and offer models, the first state had a coefficient of 1 on the previous liquidity, and a small residual standard deviation. This state is consistent with no public or private information being incorporated into prices, and little in the market changing.

The second state, which has a higher residual variance, exhibits evidence of changing liquidity. However, the coefficient on previous liquidity, and the intercept are significantly different between the two models. Accordingly, state 2 may be driven by liquidity changing for various reasons. These results motivate a 3-state model where we differentiate the state with changing liquidity into two states-one representing changing liquidity due to HFT activity:

- $\quad$ Stable liquidity

- $\quad$ Normal changing liquidity

- $\quad$ Changing liquidity due to HFT

Bid:

$$
L i q_{t}= \begin{cases}0.00+1.00 L i q_{t-1}+0.09 \Delta B A M+\epsilon_{1}, & \epsilon_{1} \sim N(0,0.002) \\ -0.83+0.49 L i q_{t-1}-0.06 \Delta B A M+\epsilon_{2}, & \epsilon_{2} \sim N(0,0.470)\end{cases}
$$

Offer:

$$
L i q_{t}=\left\{\begin{array}{cc}
0.42+1.33 L i q_{t-1}-0.12 \Delta B A M+\epsilon_{1}, & \epsilon_{1} \sim N(0,0.420) \\
0.00+1.00 L i q_{t-1}+0.16 \Delta B A M+\epsilon_{2}, & \epsilon_{2} \sim N(0,0.001)
\end{array}\right.
$$

\subsubsection{Three States}

The first state in the 3-state model again exhibits stable liquidity. The following two states exhibit varying volatility which is driven by different factors. In state 2 liquidity is driven by a change in the bid-ask midpoint. This is consistent with liquidity provision in reaction to a movement in the market - possibly driven by new information.

In state 3, however, a change in the bid-ask midpoint has no effect on liquidity. Similarly, previous liquidity explains only a quarter to a third of present liquidity, and the variance of the residual is the highest in state 3. If there is HFT activity present, it is most likely within state 3. Note, these results are consistent across both bids and asks. Lastly, we'll estimate the parameters of a 4-state model to see if state 3 is a composite of other states. 
Bid:

$$
L i q_{t}=\left\{\begin{array}{cc}
-0.00+1.00 L i q_{t-1}-0.12 \Delta B A M+\epsilon_{1}, & \epsilon_{1} \sim N(0,0.004) \\
-0.09+0.22 L i q_{t-1}+1.02 \Delta B A M+\epsilon_{1}, & \epsilon_{1} \sim N(0,0.292) \\
-0.01+0.32 L i q_{t-1}+0.004 \Delta B A M+\epsilon_{1}, & \epsilon_{1} \sim N(0,0.400)
\end{array}\right.
$$

Figure 1: Two state Markov-Switching model of liquidity available at the bid 2 State Model (Bid) 2016-11-09 14:56:56/2016-11-09 14:57:04

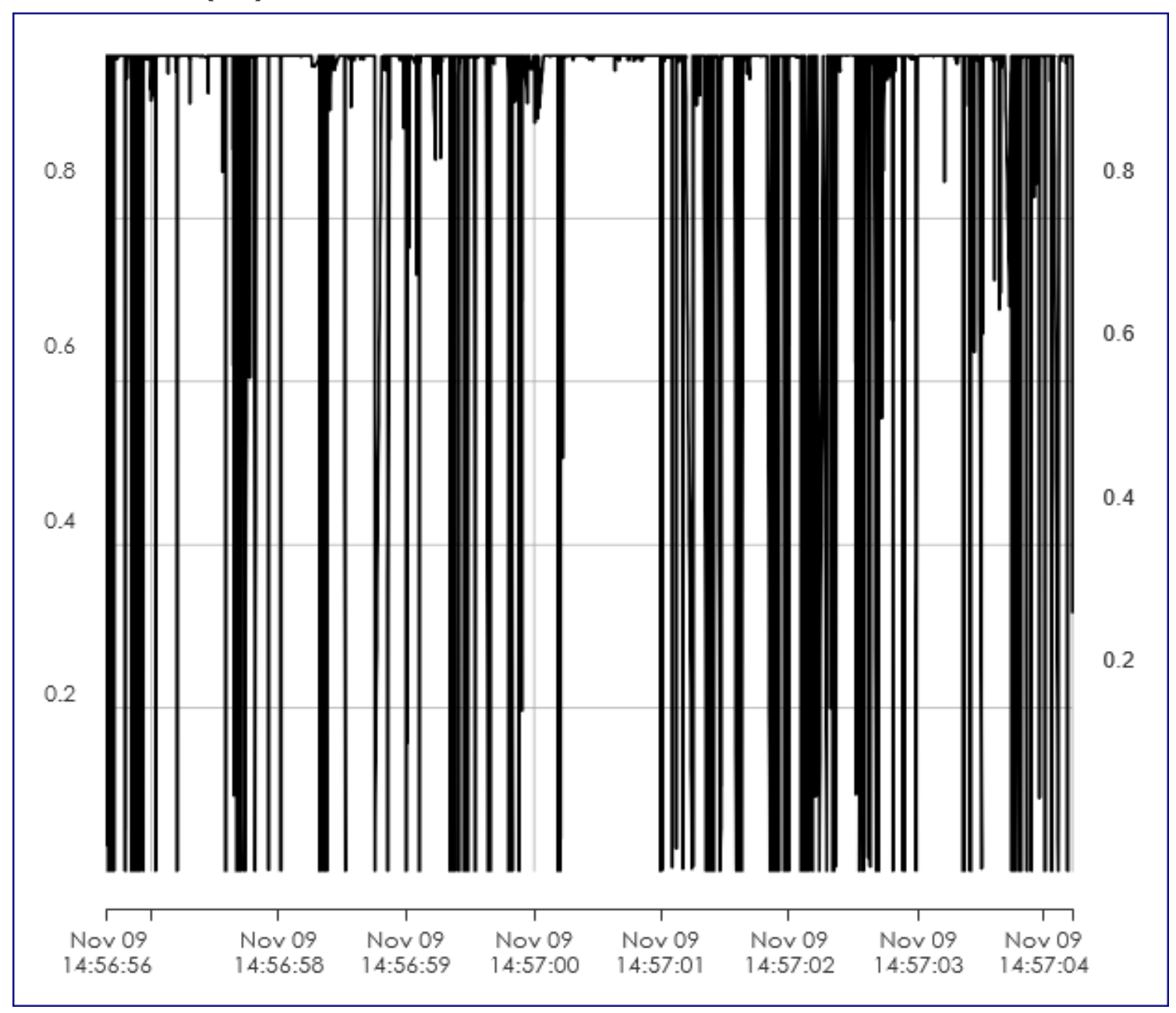


Figure 2: Two state Markov-Switching model of liquidity available at the offer 2 State Model (Offer) 2016-11-09 14:56:56 / 2016-11-09 14:57:04

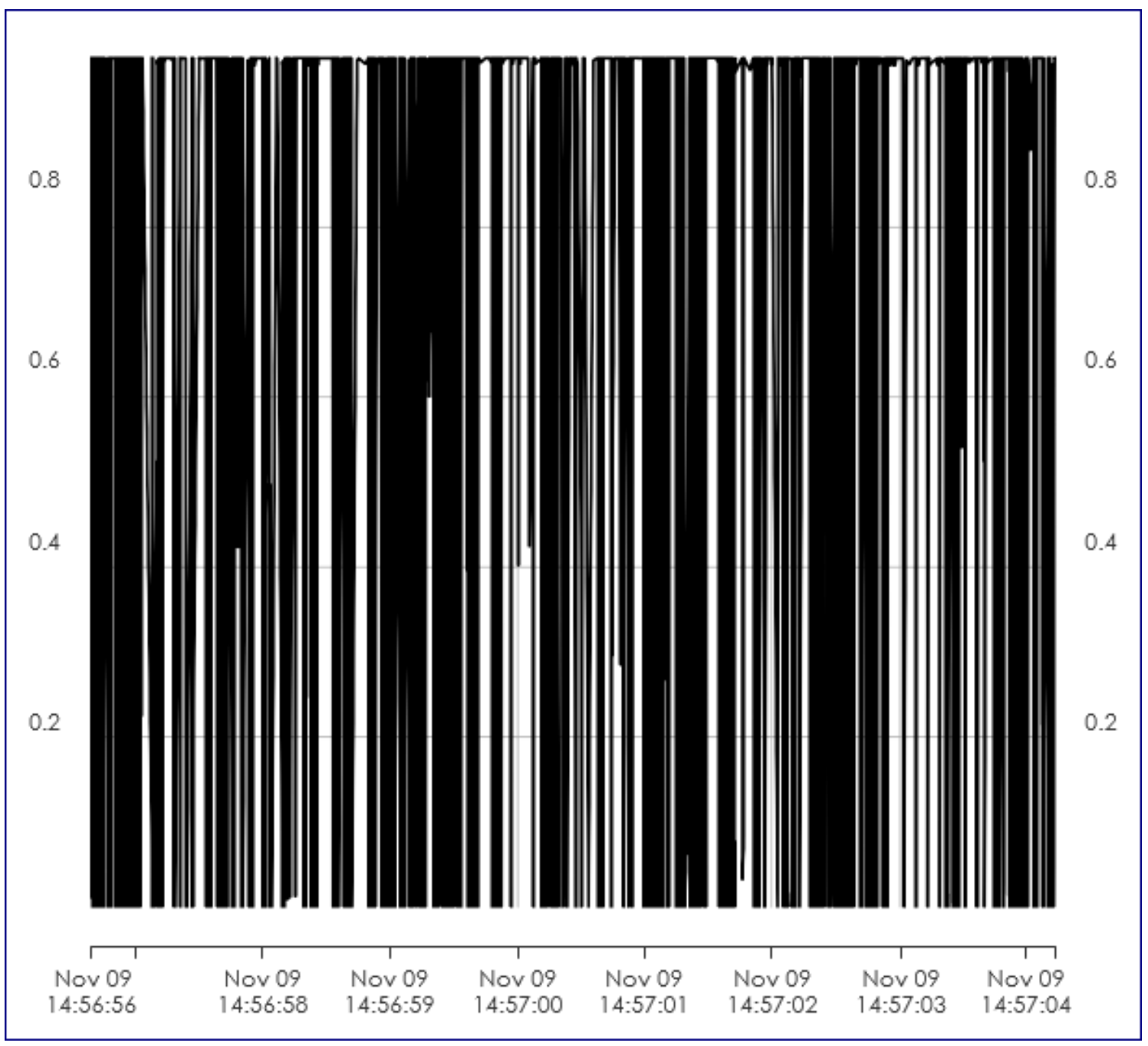


Figure 3: Three state Markov-Switching model of liquidity available at the bid

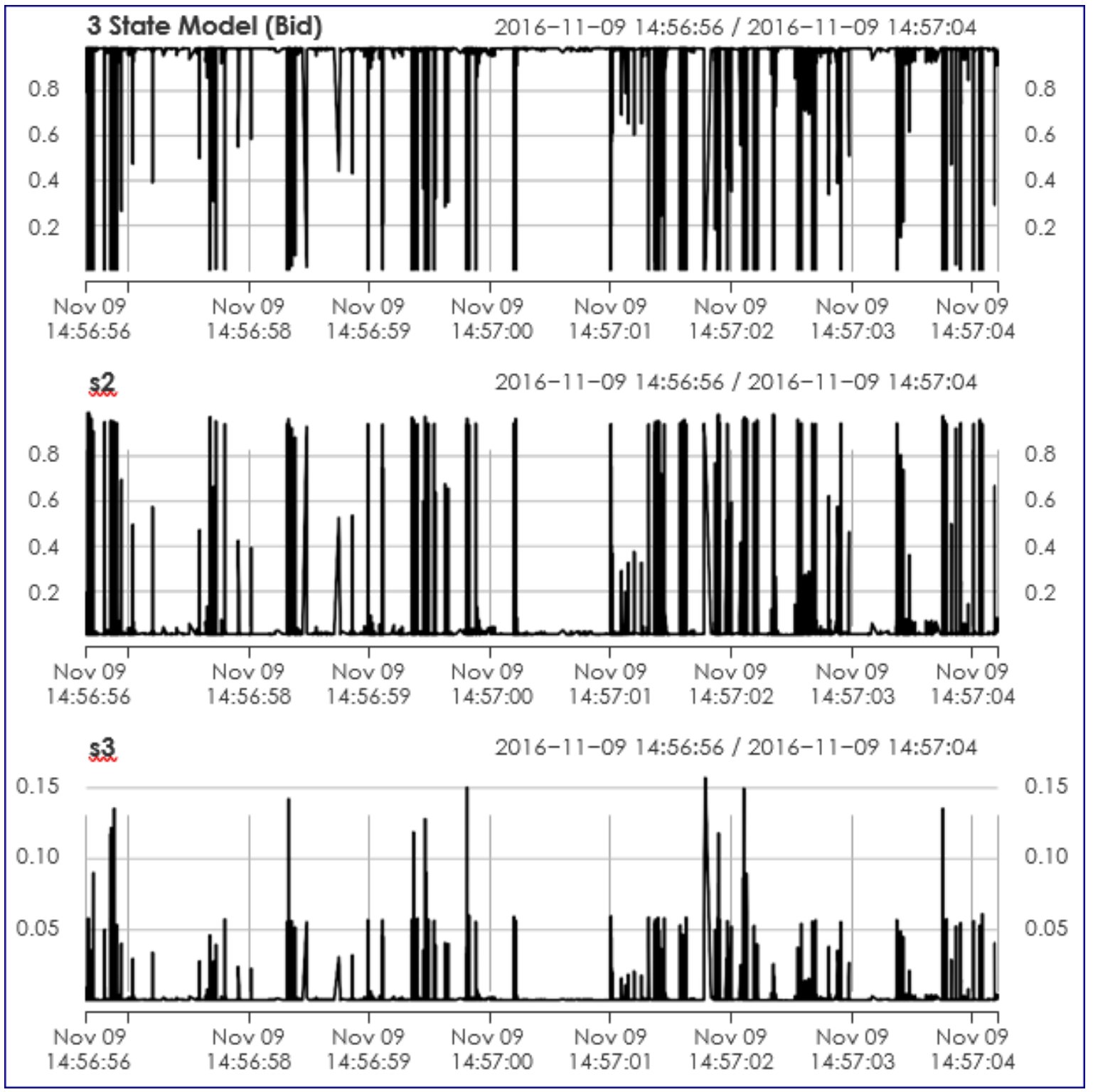

Offer:

$$
L i q_{t}=\left\{\begin{array}{cc}
-0.00+1.00 L i q_{t-1}-0.10 \Delta B A M+\epsilon_{1}, & \epsilon_{1} \sim N(0,0.004) \\
0.38-0.03 L i q_{t-1}+0.81 \Delta B A M+\epsilon_{1}, & \epsilon_{1} \sim N(0,0.078) \\
0.12+0.25 L i q_{t-1}+0.01 \Delta B A M+\epsilon_{1}, & \epsilon_{1} \sim N(0,0.900)
\end{array}\right.
$$


Figure 4: Three state Markov-Switching model of liquidity available at the offer

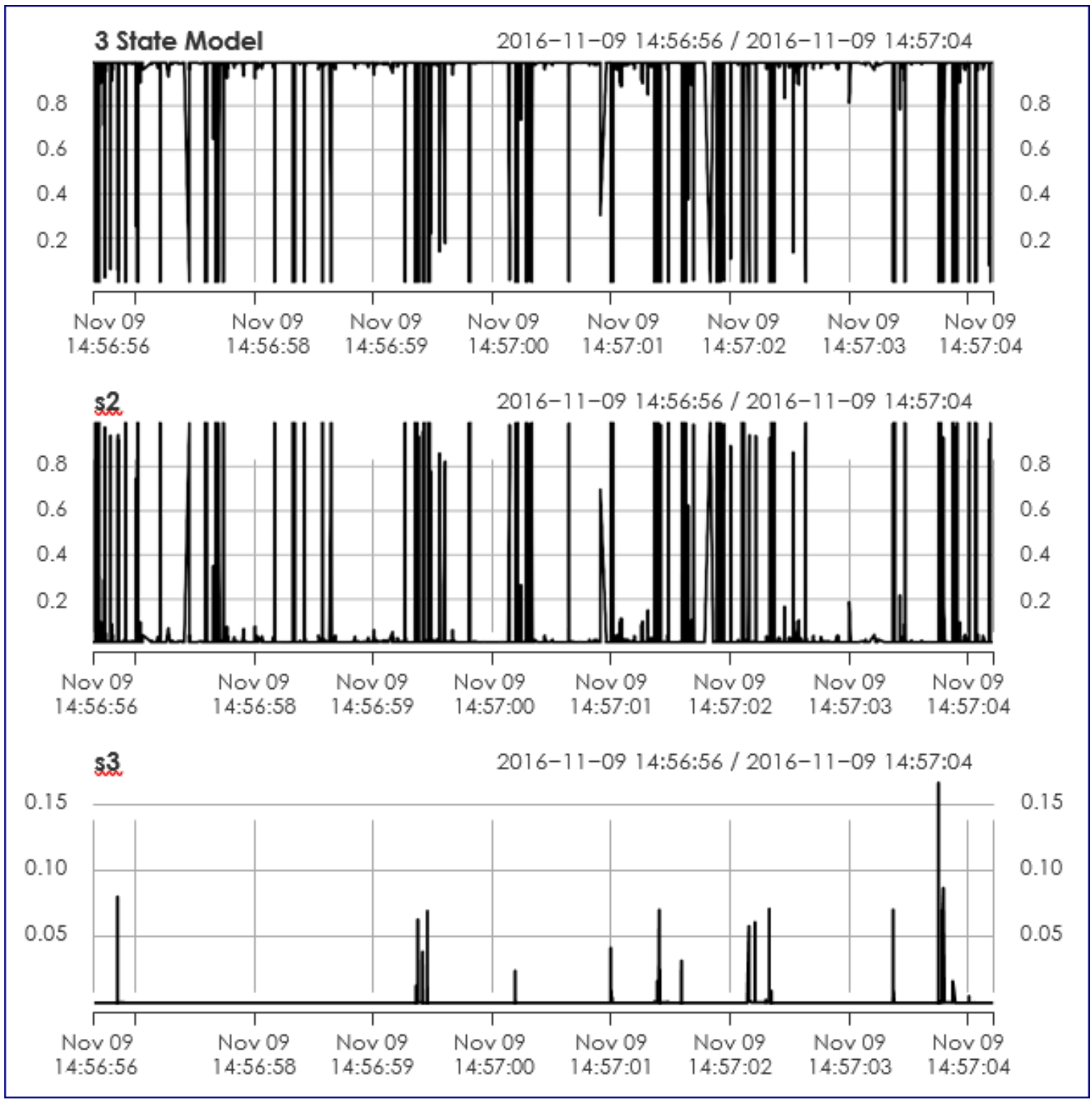

\subsubsection{Four States}

Similar to the three-state equation, the first two states represent stable liquidity, and changing liquidity driven by changes in the bid-ask midpoint. State 3 exhibits negative relationships between previous and present liquidity. The standard deviation of the error term is moderately high in this state, however it is about a quarter to a third of the standard deviation of the error term in state 4.

State 4 is most consistent with the type of HFT activity we are trying to identify. In state 4 liquidity remains constant with substantial variability around the stable mean liquidity amount. 
Bid:

$$
L_{i q} q_{t}=\left\{\begin{array}{cc}
0.0024+0.9983 \text { Liq }_{t-1}+0.1319 \Delta B A M+\epsilon_{1}, & \epsilon_{1} \sim N(0,0.0077) \\
-0.0594-0.3211 \text { Liq }_{t-1}+0.8524 \Delta B A M+\epsilon_{1}, & \epsilon_{1} \sim N(0,0.2901) \\
0.3796-0.0636 \text { Liq }_{t-1}-0.1802 \Delta B A M+\epsilon_{1}, & \epsilon_{1} \sim N(0,0.2409) \\
-0.1626+0.9469 \text { Liq }_{t-1}+0.0791 \Delta B A M+\epsilon_{1}, & \epsilon_{1} \sim N(0,0.6580)
\end{array}\right.
$$

Offer:

$$
L i q_{t}= \begin{cases}0.0000+1.0000 \mathrm{Liq}_{t-1}-0.2681 \Delta B A M+\epsilon_{1}, & \epsilon_{1} \sim N(0,0.0000) \\ -0.0055+0.9949 \mathrm{Liq}_{t-1}-1.1200 \Delta B A M+\epsilon_{1}, & \epsilon_{1} \sim N(0,0.0153) \\ -1.1325-03480 \mathrm{Liq}_{t-1}-0.0122 \Delta B A M+\epsilon_{1}, & \epsilon_{1} \sim N(0,0.1400) \\ -0.0048+1.0051 \mathrm{Liq}_{t-1}-0.5034 \Delta B A M+\epsilon_{1}, & \epsilon_{1} \sim N(0,0.6207)\end{cases}
$$

Given the above estimates, and using 0.2 as our signal threshold for state 4, the signal fires, on average, 0.636 times per second on the bid side of the orderbook. On the ask side of the orderbook the signal fires 10.59 times per second on average. Assuming a 10 millisecond delay each time the signal fires, this implies the signal duration of $0.636 \%$ and $10.59 \%$ of the trading day on the bid and ask side of the orderbook respectively. This duration range is reasonable given anecdotal accounts of the pervasiveness of high-frequency trading in markets, such as Hendershott, Jones, and Menkveld (2011) which reported that as much as $73 \%$ of volume in US markets was due to high-frequency trading.

In tables 3 and 4 in the appendix we provide parameter estimates for the 4-state model, along with signal duration estimates, for the entire week (November 7 through 11, 2016). The parameter estimates are very similar across days for each side of the orderbook. Further the signal durations are also similar with the exception of the offer side of the book on the November 9th trading day. The large release of public information occurred on November 9th, and this orderbook asymmetry with regards to algorithmic activity is consistent with Hasbrouck (2018).

\section{Table 1: Bid side of the orderbook.}

Below are coefficient estimates from the Markov-switching regressions. The standard errors are next to the coefficient in parentheses. The coefficients were estimated using the nanosecond time-stamped orderbook ranging from 6:00 PM EST on November 8, 2016 to 5:00 PM EST on November 9, 2016. There are 9,965,673 changes to the orderbook for this period.

\begin{tabular}{lccc}
\hline Coefficient & Two-State & Three-State & Four-State \\
\hline$a_{1}$ & $0.00(0.0000)$ & $-0.00(0.0000)$ & $0.00(0.0000)$ \\
$a_{2}$ & $-0.83(0.0007)$ & $-0.09(0.0250)$ & $-0.05(0.0033)$ \\
$a_{3}$ & & $-0.01(0.0140)$ & $0.37(0.0025)$ \\
$a_{4}$ & & & $-0.16(0.0018)$ \\
$\beta_{11}$ & $1.00(0.0000)$ & $0.99(0.0000)$ \\
$\beta_{12}$ & $1.00(0.0000)$ & $-0.12(0.259)$ & $0.13(0.4226)$ \\
$\beta_{21}$ & $0.09(0.1369)$ & $0.22(0.003)$ & $-0.32(0.0110)$ \\
$\beta_{22}$ & $0.49(0.0004)$ & $1.02(0.670)$ & $0.85(1.1350)$ \\
$\beta_{31}$ & $-0.06(0.0075)$ & $0.32(0.000)$ & $-0.06(0.0050)$ \\
$\beta_{32}$ & & $0.00(0.0000)$ & $-0.18(0.8833)$ \\
\hline
\end{tabular}




\begin{tabular}{lccc}
\hline Coefficient & Two-State & Three-State & Four-State \\
\hline$\beta_{41}$ & & & $0.94(0.0001)$ \\
$\beta_{42}$ & & $0.07(0.0933)$ \\
$\sigma_{1}$ & $0.00(0.0490)$ & $0.00(0.0661)$ & $0.00(0.0000)$ \\
$\sigma_{2}$ & $0.47(0.0002)$ & $0.29(0.0024)$ & $0.29(0.0044)$ \\
$\sigma_{3}$ & & $0.40(0.0001)$ & $0.24(0.0033)$ \\
$\sigma_{4}$ & & & $0.65(0.0008)$ \\
\hline
\end{tabular}

Table 2: Ask side of the orderbook.

Below are coefficient estimates from the Markov-switching regressions. The standard errors are next to the coefficient in parentheses. The coefficients were estimated using the nanosecond time-stamped orderbook ranging from 6:00 PM EST on November 8, 2016 to 5:00 PM EST on November 9, 2016. There are 9,965,673 changes to the orderbook for this period.

\begin{tabular}{lccc}
\hline Coefficient & Two-State & Three-State & Four-State \\
\hline$a_{1}$ & $0.42(0.0000)$ & $-0.00(0.0000)$ & $0.00(0.0000)$ \\
$a_{2}$ & $0.00(0.0041)$ & $0.38(0.0141)$ & $-0.00(0.0970)$ \\
$a_{3}$ & & $0.12(0.0196)$ & $-1.13(0.7924)$ \\
$a_{4}$ & & $-0.00(0.02269)$ \\
$\beta_{11}$ & $1.33(0.4078)$ & $1.00(0.0000)$ & $1.00(0.0083)$ \\
$\beta_{12}$ & $-0.10(0.2259)$ & $-0.26(0.1421)$ \\
$\beta_{21}$ & $-0.12(0.0059)$ & $-0.03(0.0192)$ & $0.99(0.0001)$ \\
$\beta_{22}$ & $1.00(0.0000)$ & $0.81(1.5312)$ & $1.12(0.0018)$ \\
$\beta_{31}$ & $0.16(0.0009)$ & $0.25(0.0027)$ & $-0.34(0.0990)$ \\
$\beta_{32}$ & $0.01(1.3956)$ & $-0.01(0.6147)$ \\
$\beta_{41}$ & & & $1.00(0.2876)$ \\
$\beta_{42}$ & & $-0.50(0.9778)$ \\
$\sigma_{1}$ & & $0.00(0.0000)$ & $0.00(0.0000)$ \\
$\sigma_{2}$ & & $0.07(0.0101)$ & $0.01(0.0626)$ \\
$\sigma_{3}$ & & $0.90(0.0002)$ & $0.14(0.0115)$ \\
$\sigma_{4}$ & $0.42(0.0011)$ & & $0.62(0.0004)$ \\
\hline
\end{tabular}

\section{Conclusion}

In this analysis we have used Markov-Switching regression models to identify the presence of high-frequency traders who are rapidly changing volatility. Using a model with four states, we identify a state with a stable mean liquidity, but substantial variability in liquidity around the mean. That is there is rapidly changing liquidity, which does not affect overall liquidity or the price. 
Figure 5: Four state Markov-Switching model of liquidity available at the bid

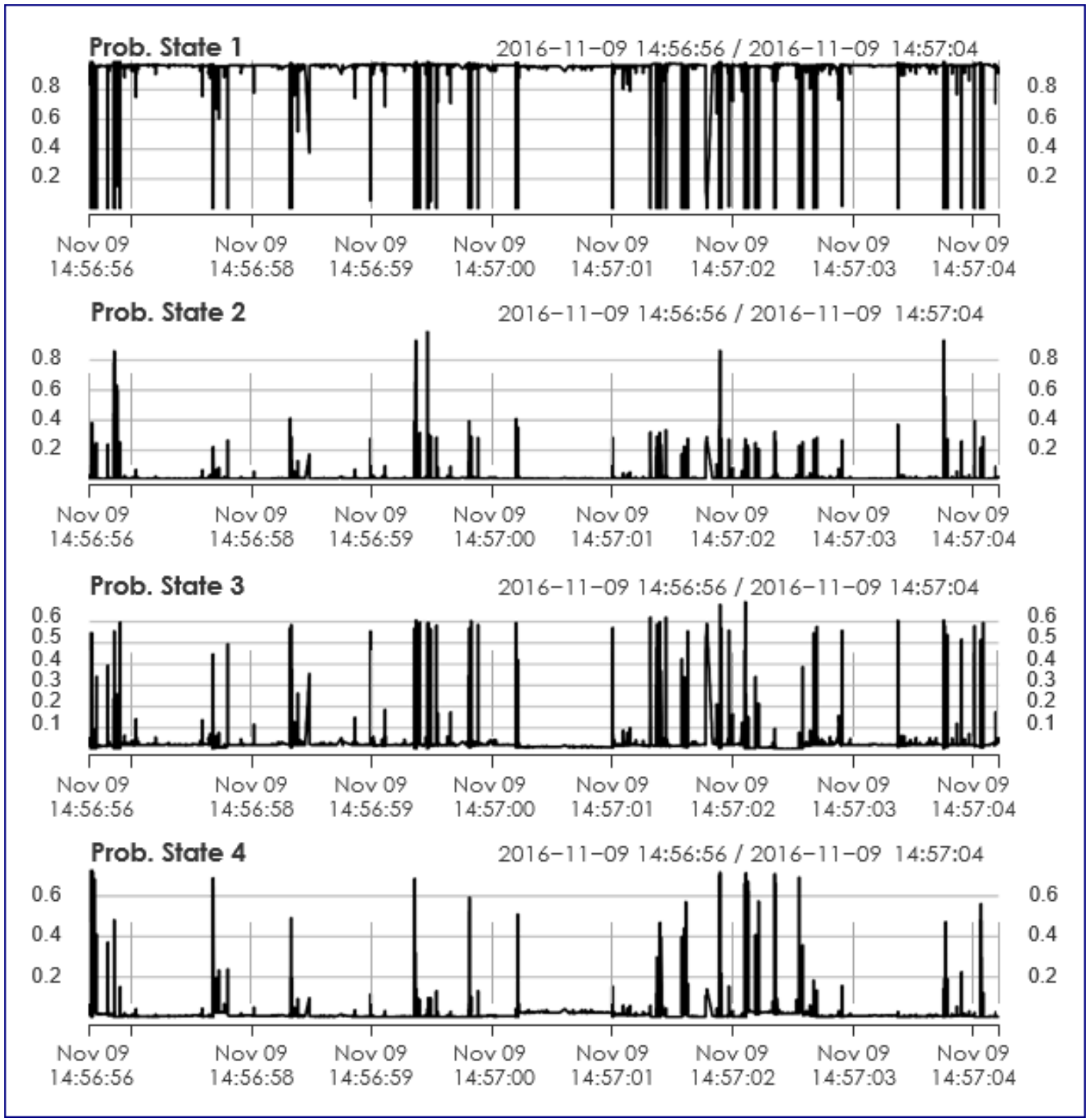


Figure 6: Four state Markov-Switching model of liquidity available at the offer

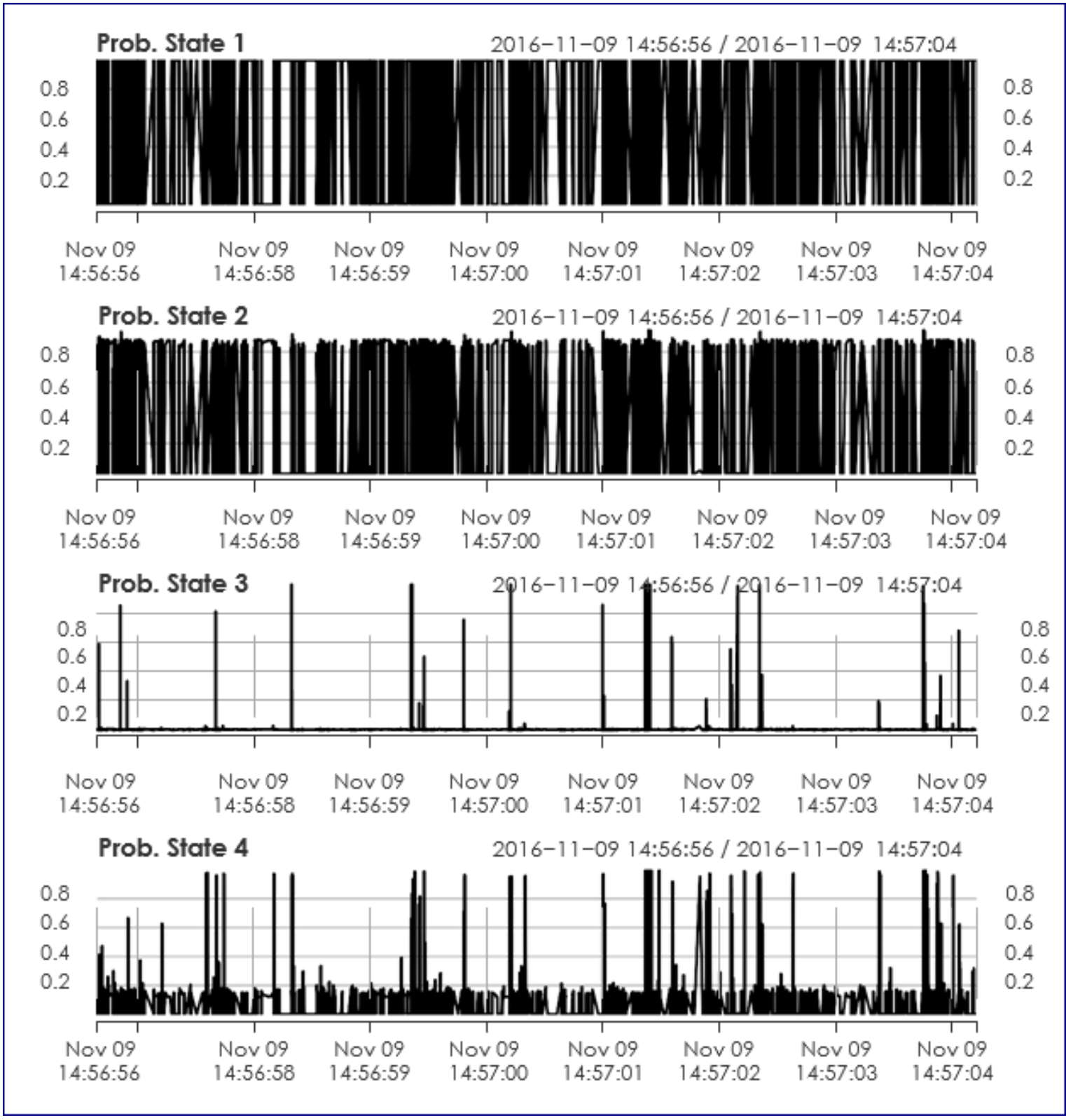

Since trading in this state benefits high-frequency traders at the expense of slower retail order flow, a transition to this state can serve as a signal to delay slower traders' orders. The delay being mere tens of milliseconds, it will not be perceptible to the typical trader. And while this may save each trade a small amount, in aggregate such a delayed order type would provide substatial savings across all non-high-frequency traders. Delaying orders due to the signal can be offered to retail traders through a particular order type. A similar strategy is used by the IEX's 'crumbling quote' order. 


\section{Appendix}

In tables 3 and 4 below are parameter estimates from the following 4-state MarkovSwitching model.

\section{Table 3: Parameter estimates from a 4-state Markov-switching model}

Parameter estimates from a 4-state Markov-switching model on the liquidity available on the bid side of the orderbook. There are 2,917,466 entries to the book over the Nov. 7 trading day. There are 3,502,097 book entries on Nov. 8. There are $9,965,673$ book entries on Nov. 9 , which is the trading day over which the results of the election were announced. There were 7,346,604 book entries on Nov. 10, and 4,905,882 on 11 November. The duration of the signal (Sig. Dur.) was calculated assuming a 10-millisecond delay for each signal, and a 0.2 threshold for the

\begin{tabular}{lccccc} 
signal generation. & \multicolumn{5}{c}{} \\
\hline Coefficient & $\mathbf{7 ~ N o v}$ & $\mathbf{8 ~ N o v}$ & $\mathbf{9 ~ N o v . ~}$ & $\mathbf{1 0 ~ N o v . ~}$ & $\mathbf{1 1 ~ N o v . ~}$ \\
\hline$a_{1}$ & 0.0065 & 0.0431 & 0.0024 & -0.0656 & -0.0010 \\
$a_{2}$ & -0.1132 & -0.1694 & -0.0594 & -0.4849 & -0.4917 \\
$a_{3}$ & 0.1121 & 0.3509 & 0.3796 & 0.3917 & 0.2975 \\
$a_{4}$ & -0.2210 & -0.1783 & -0.1626 & -0.2966 & -0.3563 \\
$\beta_{11}$ & 1.0004 & 0.8102 & 0.9983 & 0.9500 & 1.0057 \\
$\beta_{12}$ & -0.1579 & 0.0754 & 0.1319 & 0.0174 & 0.2716 \\
$\beta_{21}$ & 0.1741 & 0.0168 & -0.3211 & 0.2004 & 0.2565 \\
$\beta_{22}$ & 0.9270 & 0.8738 & 0.8524 & 0.8375 & 0.4647 \\
$\beta_{31}$ & 0.0628 & 0.1031 & -0.0636 & 0.0132 & 0.0101 \\
$\beta_{32}$ & -0.1324 & -0.1707 & -0.1802 & -0.1676 & -0.3864 \\
$\beta_{41}$ & 0.6621 & 0.6239 & 0.9469 & 0.4445 & 1.1467 \\
$\beta_{42}$ & 0.1151 & 0.0910 & 0.0791 & -0.0752 & -0.2919 \\
$\sigma_{1}$ & 0.0221 & 0.0912 & 0.0077 & 0.0109 & 0.0219 \\
$\sigma_{2}$ & 0.0920 & 0.1716 & 0.2901 & 0.4268 & 0.6963 \\
$\sigma_{3}$ & 0.1701 & 0.0787 & 0.2409 & 0.0769 & 0.1083 \\
$\sigma_{4}$ & 0.0065 & 0.0431 & 0.0024 & -0.0656 & -0.0010 \\
\hline Log Lik. & 4880164 & 117503.2 & 16693395 & 20395.45 & 249944.1 \\
Sig. Dur. & $0.736 \%$ & $0.020 \%$ & $0.636 \%$ & $0.000 \%$ & $0.000 \%$ \\
\hline
\end{tabular}

\section{Table 4: Parameter estimates from a 4-state Markov-switching model}

Parameter estimates from a 4-state Markov-switching model on the liquidity available on the offer side of the orderbook. There are 2,917,466 entries to the book over the Nov. 7 trading day. There are 3,502,097 book entries on Nov. 8 . There are $9,965,673$ book entries on Nov. 9, which is the trading day over which the results of the election were announced. There were 7,346,604 book entries on Nov. 10, and 4,905,882 on Nov. 11. The duration of the signal (Sig. Dur.) was calculated assuming a 10-millisecond delay for each signal, and a 0.2 threshold for the signal

\begin{tabular}{|c|c|c|c|c|c|}
\hline Coefficient & 7 Nov. & 8 Nov. & 9 Nov. & 10 Nov. & 11 Nov. \\
\hline al & 0.0000 & 0.0000 & 0.0000 & 0.0000 & 0.0000 \\
\hline a2 & -0.0007 & 0.0008 & -0.0055 & 0.0011 & -0.0051 \\
\hline$a 3$ & -1.1325 & -1.1374 & -1.1325 & -0.1314 & -1.1329 \\
\hline $\mathrm{a} 4$ & -0.0042 & 0.0052 & -0.0048 & -0.0060 & 0.0015 \\
\hline$\beta 11$ & 1.0054 & 1.0051 & 1.0049 & 1.0059 & 0.9979 \\
\hline$\beta 12$ & -0.2681 & -0.2707 & -0.2681 & -0.2643 & -0.0033 \\
\hline$\beta 21$ & 0.9960 & 0.9977 & 0.9949 & 0.9991 & 0.9955 \\
\hline$\beta 22$ & -1.1161 & -1.1034 & -1.1200 & -1.1195 & -1.291 \\
\hline$\beta 31$ & 0.3465 & 0.3481 & 0.3480 & 0.3487 & 0.3414 \\
\hline$\beta 32$ & -0.0121 & -0.0153 & -0.0122 & -0.0082 & -0.0064 \\
\hline$\beta 41$ & 1.2411 & 1.2369 & 1.0086 & 1.2357 & 1.0148 \\
\hline$\beta 42$ & -0.5031 & -0.5057 & -0.5034 & -0.5006 & -0.5136 \\
\hline$\sigma l$ & 0.0000 & 0.0000 & 0.0000 & 0.0000 & 0.0000 \\
\hline ఠ2 & 0.0016 & 0.0018 & 0.0153 & 0.0030 & 0.0039 \\
\hline$\sigma 3$ & 0.1400 & 0.1400 & 0.1400 & 0.1400 & 0.1400 \\
\hline$\sigma 4$ & 0.6217 & 0.6369 & 0.6207 & 0.6316 & 0.6165 \\
\hline Log Lik. & 841089.4 & 5872364 & 45918365 & 5637773 & 839429.5 \\
\hline Sig. Dur. & $0.403 \%$ & $0.797 \%$ & $10.59 \%$ & $0.704 \%$ & $1.206 \%$ \\
\hline
\end{tabular}




\section{References}

Bishop, Allison. 2017. "The Evolution of the Crumbling Quote Signal." Available at SSRN 2956535. Copeland, Thomas E., and Dan Galai. 1983. "Information Effects on the Bid-Ask Spread." The Journal of Finance 38 (5): 1457-69. https://doi.org/10.1111/j.15406261.1983.tb03834.x.

Easley, Maureen, David And O'Hara. 1992. "Time and the Process of Security Price Adjustment." The Journal of Finance 47 (2): 577-605. https://doi.org/10.1111/j.15406261.1992.tb04402.x.

French, Kenneth R., and Richard Roll. 1986. "Stock Return Variances." Journal of Financial Economics 17 (1): 5-26. https://doi.org/10.1016/0304-405x(86)90004-8.

Glosten, Lawrence R., and Paul R. Milgrom. 1985. "Bid, Ask and Transaction Prices in a Specialist Market with Heterogeneously Informed Traders." Journal of Financial Economics 14 (1): 71-100. https://doi.org/10.1016/0304-405x(85)90044-3.

Hasbrouck, Joel. 2003. "Intraday Price Formation in Us Equity Index Markets." The Journal of Finance 58 (6): 2375-2400.

"High-Frequency Quoting: Short-Term Volatility in Bids and Offers." Journal of Financial and Quantitative Analysis 53 (2): 613-41.

Hendershott, Terrence, Charles M Jones, and Albert J Menkveld. 2011. "Does Algorithmic Trading Improve Liquidity?" The Journal of Finance 66 (1): 1-33.

Kyle, Albert S. 1985. "Continuous Auctions and Insider Trading." Econometrica 53 (6): 1315. https://doi.org/10.2307/1913210.

Lee, Charles M. C., Belinda Mucklow, and Mark J. Ready. 1993. "Spreads, Depths, and the Impact of Earnings Information: An Intraday Analysis." Review of Financial Studies 6 (2): 345-74. https://doi.org/10.1093/rfs/6.2.345. 